\title{
HAWC's Eye - Implementing Hybrid Detection at the HAWC Gamma-Ray Observatory
}

\section{J. Audehm ${ }^{1, a}$, J. Serna ${ }^{2, b}$, R. Alfaro ${ }^{2}$, T. Bretz ${ }^{* 1}$, M.M. González $^{3}$, A. Iriarte ${ }^{3}$, J. Martínez-Castro $^{4}$, Y. Pérez ${ }^{3}$, M. Schaufel ${ }^{5}$ and I. Torres ${ }^{6}$ for the HAWC Collaboration}

${ }^{1}$ III. Physics Institute A, RWTH Aachen University, Germany

${ }^{2}$ Instituto de Física, Universidad Nacional Autónoma de México, Mexico

${ }^{3}$ Instituto de Astronomía, Universidad Nacional Autónoma de México, Mexico

${ }^{4}$ Centro de Investigacion en Computación, Instituto Politecnico Nacional, Mexico

${ }^{5}$ III. Physics Institute B, RWTH Aachen University, Germany

${ }^{6}$ Instituto Nacional de Óptica y Electrónica, Mexico

Email: ${ }^{a}$ jan.audehm@rwth-aachen.de, ${ }^{b}$ j_serna@ciencias.unam.mx

For a complete author list, see https://www.hawc-observatory.org/collaboration/icrc2019.php

Combining a compact imaging air-Cherenkov telescope with the High-Altitude Water Cherenkov Gamma-Ray Observatory (HAWC) promises significant improvements in energy resolution, angular resolution and background suppression. The HAWC Observatory is an extensive air-shower array located in the state of Puebla, Mexico, sensitive at TeV energies continuously monitors $2 \mathrm{sr}$ of the sky. An improved sensitivity has the power to significantly increase the number of monitored sources. Triangulation by a compact air-Cherenkov telescope in a hybrid setup overcomes ambiguities on the height of the shower core if measured only with a ground array. A Cherenkov telescope prototype featuring a $50 \mathrm{~cm}$ Fresnel lens and a camera with 61 semi-conductor photo sensors (SiPM) was commissioned and temporarily installed at the HAWC site in summer 2017. For the first time, hybrid events were recorded and the energy threshold and other basic properties of the telescope were measured and compared with simulations. Results of this campaign will be presented.

37th International Cosmic Ray Conference - ICRC2019

July - August, 2019

Madison, WI, USA

\footnotetext{
* Speaker.
} 


\section{Introduction}

Understanding all the processes that follow the creation of new galaxies or stars is still an unsolved puzzle. With the discovery of cosmic rays, a window to a new theoretical and experimental area was opened. In particular, understanding what kind of sources produce cosmic rays and which are the processes leading to extreme acceleration of particles motivated the development of advanced experimental techniques to detect gamma-rays.

Two of those techniques are ground-based water Cherenkov arrays and imaging air-Cherenkov telescopes (IACT). Each observation technique has its own limitations at reconstructing the shower parameters. A ground-based array detects the fraction of the shower that reaches the ground level. From this, the shower development can only be estimated, while an IACT can measure the development directly. As the techniques are complementary, making hybrid observations will improve the estimation of the shower parameters. In particular, the energy reconstruction, the angular resolution and the background suppression will be improved.

In this paper we present the work to combine the High-Altitude Water Cherenkov Gamma-Ray Observatory (HAWC) with the compact imaging air-Cherenkov telescope HAWC's Eye. Results from a first measurement campaign in 2017 are discussed and compared to simulation results.

\section{The High Altitude Water Cherenkov Gamma-Ray Observatory}

The HAWC Observatory is located at Sierra Negra in Mexico at $4100 \mathrm{~m}$ above sea level. It is an extended array of 300 water tanks with $7.3 \mathrm{~m}$ diameter and $4.5 \mathrm{~m}$ height that are filled with $200000 \mathrm{~L}$ of water each. Each water Cherenkov detector is instrumented with four photomultipliers tubes to detect the Cherenkov light associated to charged particles from the air showers produced by high energetic gamma rays. The HAWC Observatory has an instantaneous field of view of $\sim 2 \mathrm{sr}$ and a duty cycle of $>95 \%$. It is sensitive to gamma rays in the range of $300 \mathrm{GeV}$ to $100 \mathrm{TeV}$ [1]. Therefore, it is able to detect the emission from several kind of sources, such as Active Galactic Nuclei, Gamma-ray Bursts or Super Nova Remnants [2, 3].

\section{The HAWC's Eye Imaging air-Cherenkov Telescope}

The HAWC's Eye telescope which was tested on site is a compact and light-weighted IACT [4]. It is composed by a Fresnel lens of $549.7 \mathrm{~mm}$ diameter that focuses light to the camera located in the focal plane with a distance of $502.1 \mathrm{~mm}$ from the lens. The camera is made up of 64 semiconductor photo sensors (SiPMs) of type Hamamatsu S10985-100C; 61 of the SiPMs are equipped with an aluminum Winston cone to enlarge the effective of light collection. Each camera pixel (SiPM + Winston cone) has a field-of-view of $1.5^{\circ}$ giving a total field-of-view of $12^{\circ}$. The three pixels without light-collector, the blind pixels, are used for monitoring proposes. To seal the camera from environment stress, the pixels are enclosed in a carbon barrel [5].

The telescope data acquisition system, consisting of a pre-amplifier, a trigger unit and a digitizer, is an adaption of the FACT telescope data acquisition system (Figure 1) [6]. To manage the trigger system, the camera pixels are divided into patches of nine at the most. All signals of each pixel of the same group are summed and discriminated against a previously given trigger threshold. For 
bigger signals than the trigger threshold, the trigger unit enable the data acquisition. Then, the pixel signals are read out by DRS4 domino ring sampling chips with a sampling rate of 2 GS/s [7]. The entire HAWC's Eye telescopes costs less than 10,000 Euro, this makes this telescope well suited for extension of existing detector facilities [8].

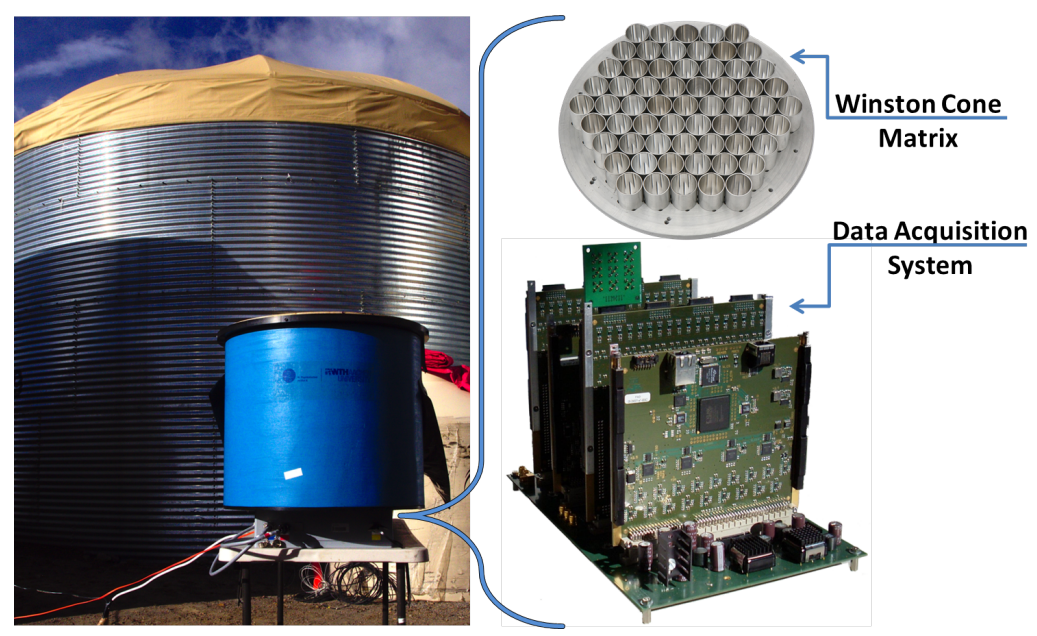

Figure 1: HAWC's Eye telescope in front of a HAWC tank during operation at the HAWC site in 2017. On the right hand side the Winston cone matrix for the camera and the data acquisition system adapted from the FACT telescope are displayed in detail. The FACT DAQ consists of two pre-amplifiers, trigger units and digitizer boards connected to a customized backplane [6].

\section{Measuring Telescope Performance Using HAWC Shower Reconstruction}

In 2017, the telescope was temporary installed and operated at the HAWC site to take first hybrid data with the HAWC Observatory. The data stream from both detectors is synchronized with the trigger time-stamps. A HAWC's Eye trigger time-stamps is stored in an empty HAWC channel if it fulfills two conditions: The trigger flag of HAWC's Eye appeared inside a given window of $1500 \mathrm{~ns}$ after a HAWC trigger and in addition more than 28 tanks of HAWC have triggered. By this procedure, one obtains two trigger patterns - one of HAWC and one of HAWC's Eye. Synchronization of the data are then achieved by shifting the patterns with respect to each other in arbitrary steps until the smallest mean time difference between corresponding trigger pairs is achieved. In the synchronized data the mean time difference is around $1 \mathrm{~ms}$, as shown in Figure 2.

Since the HAWC reconstruction is well approved and energy-, angular- as well as core resolution of the HAWC detector have been determined, the reconstructed shower parameters calculated for synchronized events by the HAWC analysis can be used to extract the telescope's benchmark parameters $[3,9,10]$. Obtained parameters as, for example, energy threshold can be compared to expectations gained from simulations.

To enhance the data quality for the analyses, two cuts are applied to the synchronized data. To get rid of random coincidences between the telescope and the array, showers that hit less than 150 HAWC tanks are removed form the sample. In addition only showers with more than 40 PMTs triggered within a radius of 40 around the reconstructed shower core are taken to account $\left(\mathrm{Nr}_{40}{ }^{-}\right.$ 


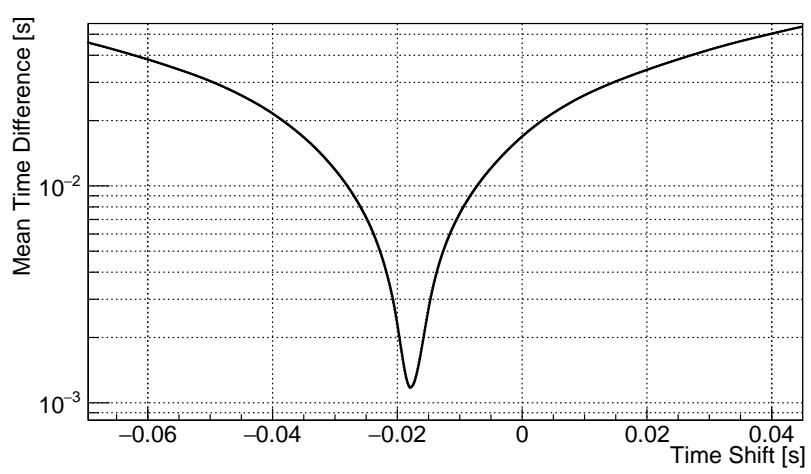

Figure 2: Synchronization quality plot for a real subrun. The mean time difference between corresponding trigger pairs is shown in dependency of the relative shift of the trigger patterns of HAWC and HAWC's Eye. A sharp minimum of the mean time difference is observed.

cut). Figure 3 displays the distribution of reconstructed shower core positions after both cuts are performed. Shower core positions are restricted to the vicinity of the HAWC tanks by the $\mathrm{Nr}_{40}$-cut. This distribution peaks near the telescope position marked by the black star and flattens radially symmetric with greater distance to it as one expects due to the limited field of view of the telescope [11].

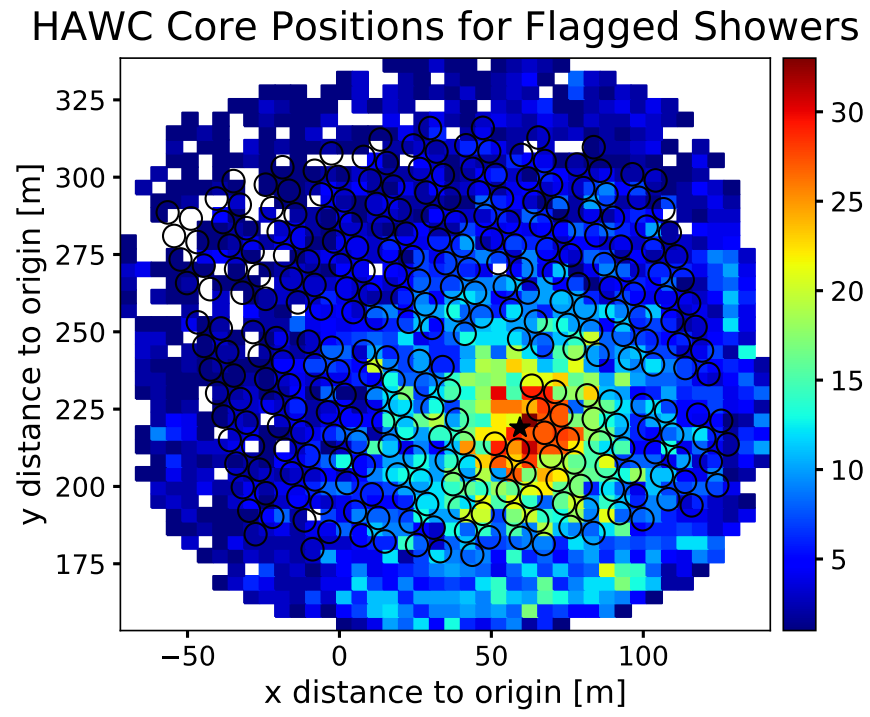

Figure 3: Histogram of shower cores positions reconstructed by HAWC for events flagged by HAWC's Eye after all cuts are applied. The core positions are given in the coordinate system of HAWC and the black star marks the location of HAWC's Eye in the HAWC detector array.

\subsection{Field of View}

The field-of-view of the telescope can be estimated to $12^{\circ}$ by considering the $1.5^{\circ}$ view cone of a single pixel. Using the HAWC reconstruction for shower arrival directions, a check of this 
expectation can be performed. When operated at the HAWC site the telescope was slightly tilted away from the HAWC zenith. As a consequence, all arrival directions reconstructed in the HAWC analysis should be rotated into the tilted telescope system before the field-of-view can be considered. Figure 4 shows a histogram of all arrival directions reconstructed in the HAWC analysis after the correction into the telescope reference system for showers that triggered HAWC's Eye. A hexagonal shaped structure originating from the shape of the camera and the imaging of showers in the optical system is visible. Inside the expected field-of-view, shower directions are distributed homogeneously in zenith and azimuth. While outside the field of view, the number of detected showers drops off rapidly as expected.

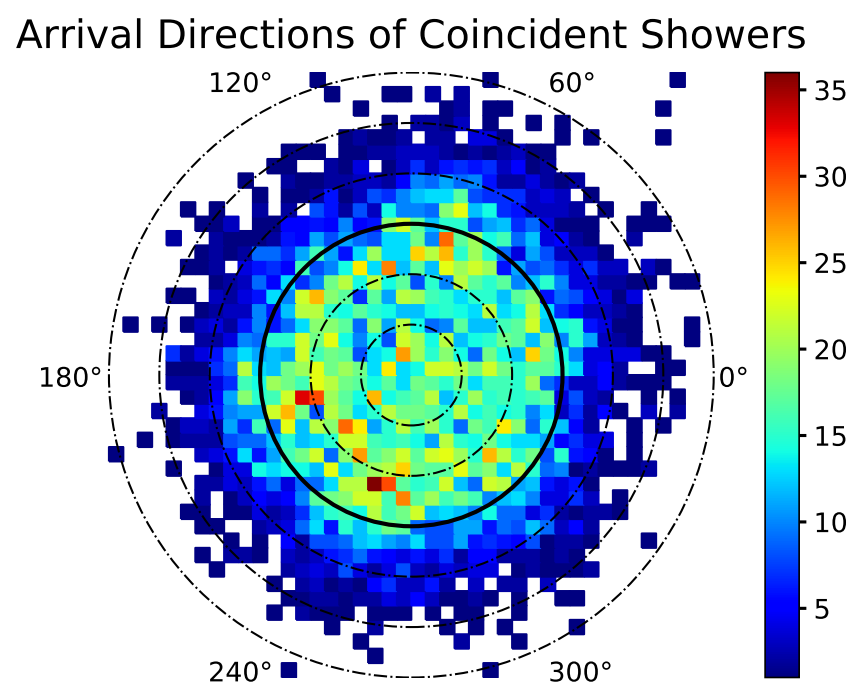

Figure 4: Arrival directions of the events detected by HAWC's Eye displayed in the telescope system. The hexagonal shape of the camera can also be recognized. Azimuth (displayed as angle) and zenith (displayed as radius) angle reconstructed by the HAWC analysis and corrected into the telescope system. Concentric circles are drawn in steps of two $2^{\circ}$ in zenith angle. The solid circle marks the field of view of the telescope. The performance is as expected.

\subsection{Effective Collection Area}

An important performance parameter for an IACT describing the detectable flux of cosmic rays is the effective collection area. Usually, simulation studies are needed to calculate this parameter. In the case, when the telescope is operated in coincidence with the HAWC observatory, geometrical shower information from HAWC offer the possibility to directly measure the effective area of HAWC's Eye instead of relaying exclusively on simulations.

Considering only showers with a zenith angle $<5.5^{\circ}$, i.e. within the region of constant telescope response, the active area of the telescope can be calculates by dividing the number of coincident showers by the total number of showers observed by HAWC and multiplying this with the accounted area. In this calculation the HAWC detector angular response is assumed to be flat in this zenith region, so that no further response correction is needed. 
Here for the calculation of the effective area three different regions were considered. Two of them should in addition only contain showers with a shower core inside the instrumented area of HAWC. For those regions the shower core to telescope distances are restricted to a maximum $\left(r_{0}\right)$ equal to $95 \mathrm{~m}$ and $120 \mathrm{~m}$ respectively. A third region with a maximum shower core to telescope distance of $250 \mathrm{~m}$ includes in addition shower cores located outside the detector array. If the restriction on the core distance to the telescope causes the accountable area to be a sector of a full circle, the calculated effective area is scaled to a full circle with radius $r_{0}$ afterwards. Results from this calculation are shown in Figure 5 for energies from $1 \mathrm{TeV}$ to $\sim 3.2 \mathrm{PeV}$. As a comparison, the preliminary result of a first simulation of the HAWC's Eye effective area for a patch threshold of 110 P.E. is displayed as well. Comparing the simulation with the measurements, two effects are visible: On one hand, the effective area is underestimated by the simulation as can be seen for energies between $10 \mathrm{TeV}$ and $100 \mathrm{TeV}$. On the other hand the simulation reproduces the actual trigger threshold of the telescope well and the cut of at lower energies is compatible with the measurements. The measurements for the different accounted areas themselves give consistent results. For all of them the effective area saturates at an effective area given by $\pi \cdot r_{0}^{2}$ where $r_{0}$ is the accounted radius chosen in advance. This results for a distance of $250 \mathrm{~m}$ to the telescope in a effective area of $>10^{5} \mathrm{~m}^{2}$. This is comparable to other IACTs [11].

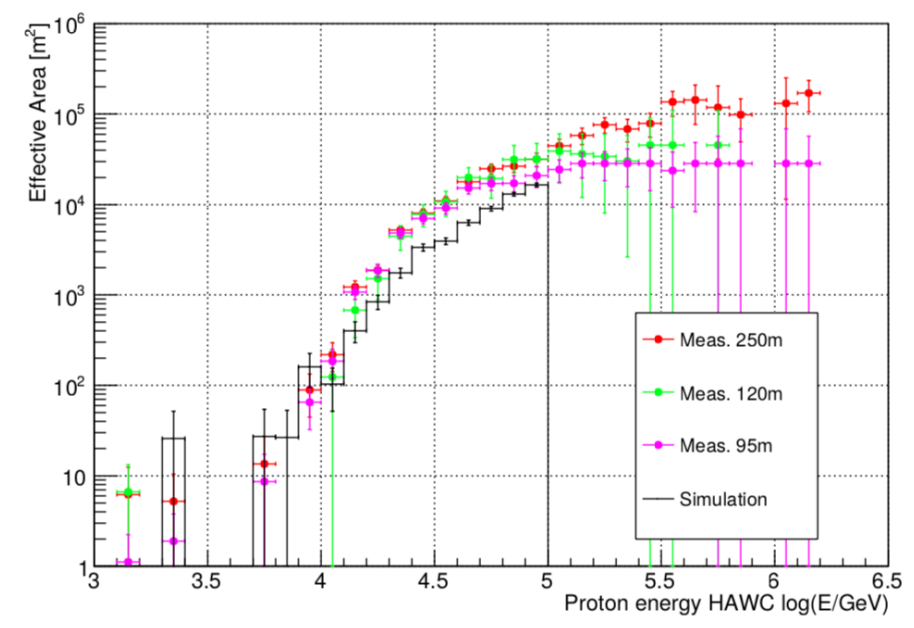

Figure 5: Measurement of the effective area of HAWC's Eye for the consideration of three different areas addressed (red, green, pink data points) together with the simulation result for a threshold of 110 P.E (black solid) [11].

\subsection{Energy Threshold}

The telescope's energy threshold for protons can be measured by accounting the proton energy reconstruction of HAWC. If the distribution of reconstructed energies is weighted by energy and divided by measurement time the proton flux is obtained (see Figure 6 on the left). This flux drops rapidly at the energy threshold of the telescope due to a decreasing detection efficiency below the threshold energy. By determining the maximum flux the energy threshold of HAWC's Eye can be determined to $(22 \pm 1.5) \mathrm{TeV}$ [11].

To those distributions a first simulation is adjusted after correction for the response of the HAWC 
detector as shown in Figure 6 on the left hand side. The simulated and measured curve are congruent for proton energies greater $12.5 \mathrm{TeV}$. An identical detector simulation is applied to gamma Monte Carlo data with a slope of -2 . Extracting the peak of the gamma flux distribution by fitting a log-normal function to the peak area, the expected energy threshold for gamma primaries is $\sim 10 \mathrm{TeV}$. This result is well consistent with a lower threshold by a factor two to three as expected from shower physics (see Figure 6 right hand side) [12].

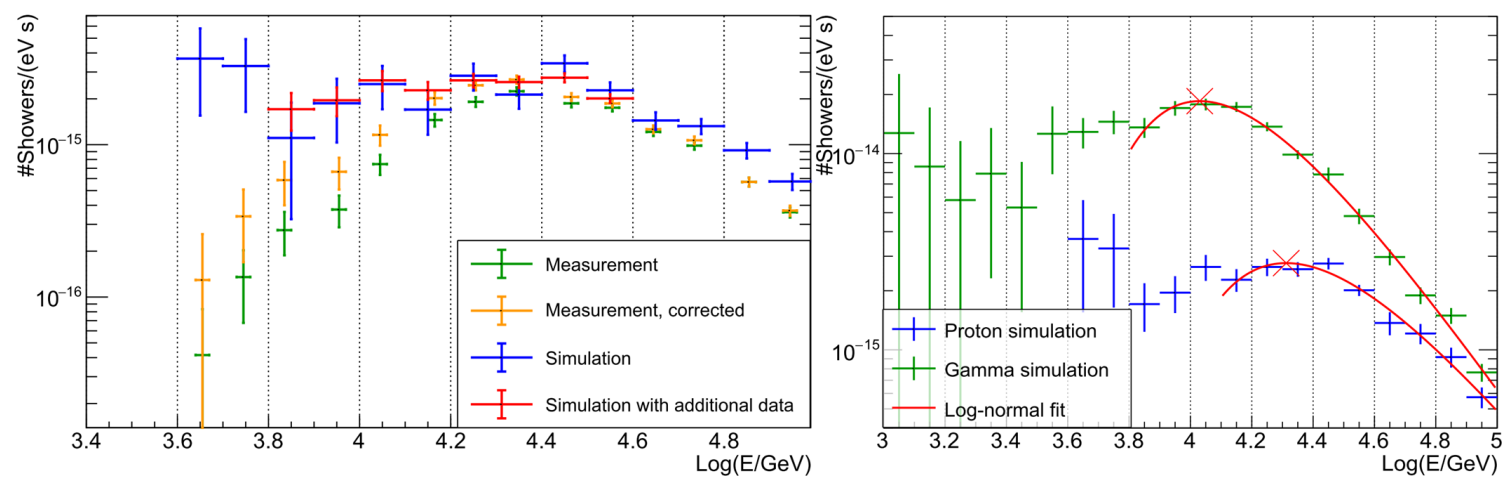

Figure 6: Energy threshold estimated for HAWC's Eye. Left: Comparison between the measured flux of protons and simulations of the same quantity. The measured curve is given before (green) and after (yellow) the correction for the HAWC response curve. Two simulations are given (blue, red). Right: Estimation of the energy threshold for protons (blue) and photons (green) using Monte Carlo simulations. For each curve a log-normal function is fitted to the peak area to estimate the threshold [12].

\section{Summary and Outlook}

During a first measurement campaign a HAWC's Eye telescope was operated at the HAWC Gamma-Ray Observatory. Together with the HAWC detector hybrid data was taken and synchronized successfully. The HAWC Gamma-Ray Observatory and the HAWC's Eye telescope succeeded in taking hybrid IACT and ground-based array data, allowing for the first time to characterize and calibrate the IACT without the need of simulations. A detector simulation was tuned to coincide with the obtained parameters and consequently used to derive the energy threshold for gamma-rays to be located around $10 \mathrm{TeV}$.

Currently, preparations and last test for an installation of two improved HAWC's Eye telescopes at the HAWC site are ongoing. The update includes improved hexagonal light-collectors made of Polymethyl methacrylate (PMMA) as well as a change to silicon photomultipliers from the SensL MicroFJ-600xx-SMT series which have improved performance. Both improvements together promise an increase in light collection efficiency by more than a factor of three. Installed at the site the telescopes will enable recording first stereoscopic hybrid data taken by a ground based detector array and two telescopes and a confirmation of the expected improvement of the energy reconstruction, the angular resolution and the background suppression. First measurements are expected to take place not later than August. 


\section{Acknowledgments}

We acknowledge the support from: the US National Science Foundation (NSF); the US Department of Energy Office of High-Energy Physics; the Laboratory Directed Research and Development (LDRD) program of Los Alamos National Laboratory; Consejo Nacional de Ciencia y Tecnología (CONACyT), México (grants 271051, 232656, 260378, 179588, 254964, 271737, 258865, 243290, 132197, 281653) (Cátedras 873, 1563, 341), Laboratorio Nacional HAWC de rayos gamma; L'OREAL Fellowship for Women in Science 2014; Red HAWC, México; DGAPA-UNAM (grants AG100317, IN111315, IN111716-3, IA102715, IN111419, IA102019, IN112218), VIEP-BUAP; PIFI 2012, 2013, PROFOCIE 2014, 2015; the University of Wisconsin Alumni Research Foundation; the Institute of Geophysics, Planetary Physics, and Signatures at Los Alamos National Laboratory; Coordinación de la Investigación Científica de la Universidad Michoacana; Polish Science Centre grant DEC-2014/13/B/ST9/945, DEC-2017/27/B/ST9/02272; Royal Society Newton-Advanced Fellowship 180385. Thanks to Scott Delay, Luciano Díaz and Eduardo Murrieta for technical support.

We acknowledge as well: the Verbundforschung of the German Ministry for Education and Research (BMBF), funded by the Excellence Initiative of the German federal and state governments and the Helmholtz Alliance for Astroparticle Physics (HAP); the German Academic Exchange Service (DAAD); the FACT collaboration and the Pierre Auger Observatory, as well as the HAWC and IceCube Collaborations; the mechanical and electronic workshops of the RWTH Aachen University for their permanent support.

\section{References}

[1] Smith, A.J. et al. [HAWC Collaboration], HAWC: Design, Operation, Reconstruction and Analysis, In Proc. of the $34^{\text {th }}$ ICRC [PoS(ICRC2015)966].

[2] Abeysekara, A.U. et al. [HAWC Collaboration], Sensitivity of the High Altitude Water Cherenkov detector to sources of multi-TeV gamma rays, The Astrophysical Journal 50-52 (2013) [doi:10.1016/j.astropartphys.2013.08.002].

[3] Abeysekara, A.U. et al. [HAWC Collaboration], The 2HWC HAWC Observatory Gamma Ray Catalog, The Astrophysical Journal 843 (2017) [doi:10.3847/1538-4357/aa7556].

[4] Bretz, T. et al., A compact and light-weighted refractive telescope for the observation of extensive air showers, JINST 13 (2018) P07024 [doi:10.1088/1748-0221/13/07/P07024].

[5] Niggemann, T. et al., Status of the Silicon Photomultiplier Telescope FAMOUS for the Fluorescence Detection of UHECRs, In Proc. of the $33^{\text {rd }}$ ICRC [arXiv:1502.00792].

[6] Anderhub, H. et al. [FACT Collaboration], Design and Operation of FACT - The First G-APD Cherenkov Telescope, JINST 8 (2013) P06008 [doi:10.1088/1748-0221/8/06/P06008].

[7] Ritt, S., Design and performance of the $6 \mathrm{GHz}$ waveform digitizing chip DRS4, In Nuclear Science Symposium Conference Record, 2008 [doi:10.1109/NSSMIC.2008.4774700].

[8] Auffenberg, J. et al, Design study of an air-Cherenkov telescope for harsh environments with efficient air-shower detection at $100 \mathrm{TeV}$, In Proc. of the $34^{\text {th }}$ ICRC [PoS(ICRC2015)1156].

[9] Abeysekara, A.U. et al. [HAWC Collaboration], Observation of the Crab Nebula with the HAWC Gamma-Ray Observatory, The Astrophysical Journal 843 (2017) [doi:10.3847/1538-4357/aa7555].

[10] Alfaro, R. et al. [HAWC Collaboration], All-particle cosmic ray energy spectrum measured by the HAWC experiment from 10 to $500 \mathrm{TeV}$, Physical Review D 96 (2017) [doi:10.1103/PhysRevD.96.122001].

[11] Schaufel, M., HAWC's Eye: Implementing hybrid detection by combining a compact air-Cherenkov telescope with the HAWC Gamma-Ray Observatory. Master's Thesis, RWTH-Aachen University, 2017.

[12] Schalz, M., Energy Threshold of the compact Imaging Air-Cherenkov Telescope HAWC's Eye. Bachelor's Thesis, RWTH-Aachen University, 2017. 